\title{
A seminal vesicle cyst palpable in the rectum
}

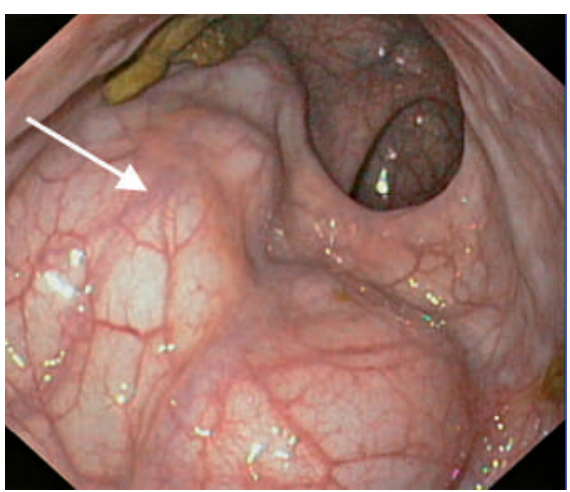

Figure 1 Sigmoidoscopic view showing multiple round impressions covered by normal colon mucosa ventrally in the distal rectum (arrow).

A 17-year-old boy presented with a history of chronic recurrent abdominal pain in the umbilical region from the age of 13 years. His bowel habit was unremarkable and he had had no rectal bleeding or difficulty in passing urine. On digital rectal examination, a smooth, firm, multilobular mass was palpable. Extensive laboratory investigations (of blood, stool, and urine samples, including urine cultures) showed no abnormalities. Sigmoidoscopy revealed multiple round impressions covered by normal colon mucosa ventrally in the distal rectum (Figure 1). Endoscopic ultrasound showed multiple echo-poor lesions, compatible with cysts, cranial and caudal to the prostate (Figure 2 ). Computed tomography showed a left-sided mass, $8 \times 4 \mathrm{~cm}$ in size, between the bladder and the rectum, an absent left kidney, and an asymmetrical bladder (Figure 3).

Laparoscopic exploration was performed and revealed a cyst of the seminal vesicle. The cyst, which contained $250 \mathrm{ml}$ of fluid, was removed. Eight months after the laparasopic resection, no new episodes of abdominal pain have occurred and the patient is doing well. Seminal vesicle cysts are uncommon and are associated with anomalies of the ipsilateral mesonephric duct [1].
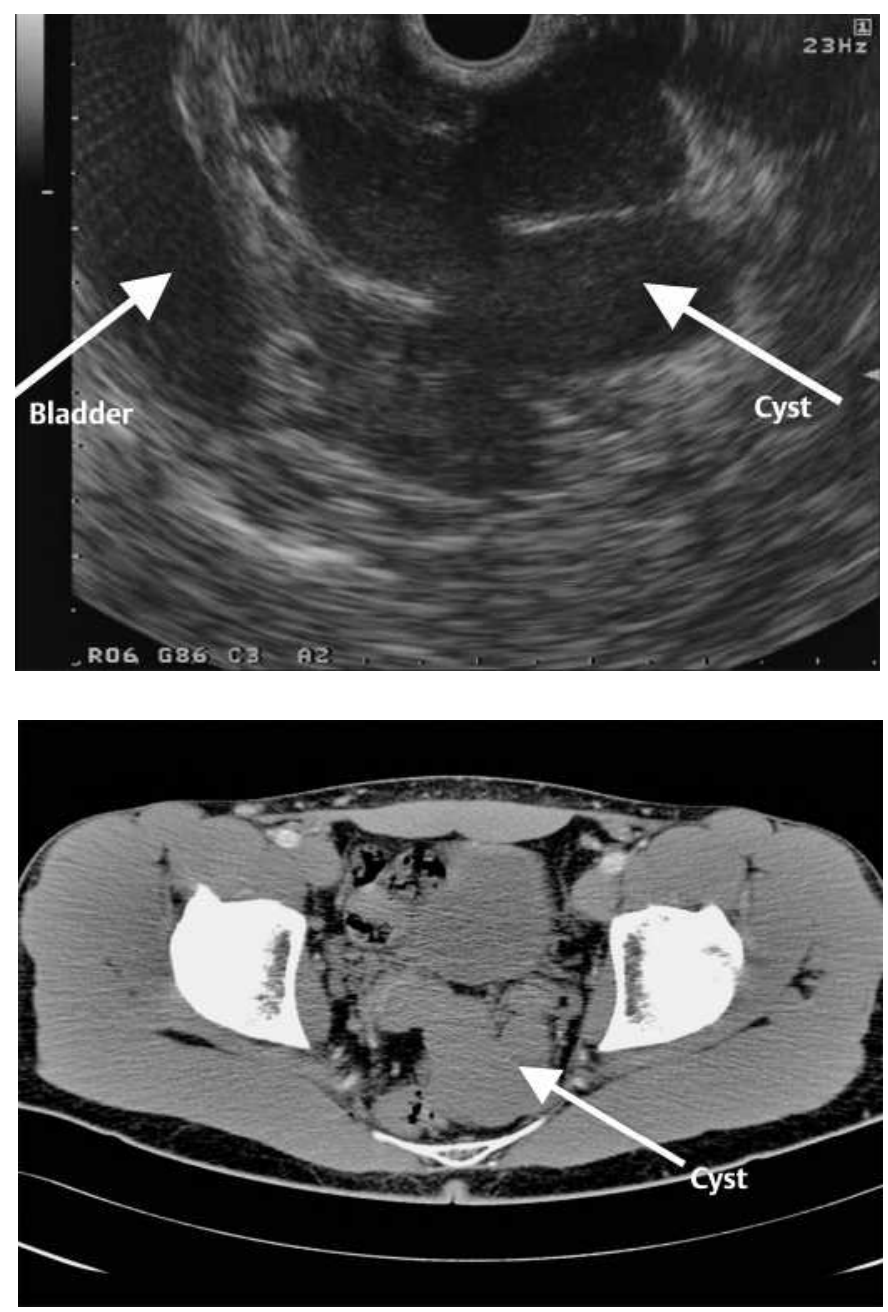

Figure 3 Computed tomographic scan showing a leftsided mass between the bladder and the rectum. The left kidney was absent and the bladder was asymmetrical.

M. A. van Meegen', F. Kokke², P. Dik², P. Fockens ${ }^{3}$, M. A. Benninga ${ }^{1}$

${ }^{1}$ Emma Children's Hospital, Academic Medical Center, Amsterdam, The Netherlands

2 Children's Hospital, University Medical Center Utrecht, Utrecht, The Netherlands

${ }^{3}$ Department of Gastroenterology and Hepatology, Academic Medical Center, University of Amsterdam, The Netherlands.

\section{References}

${ }^{1}$ King BF, Hattery RR, Lieber MM et al. Congenital cystic disease of the seminal vesicle. Radiology 1991; 178: 207
Corresponding Author

\section{A. Benninga, M.D.}

Emma Children's Hospital Academic Medical Centre G8-205

Meibergdreef 9

1105 AZ Amsterdam

The Netherlands

Fax: +31-20-6917735

E-mail: m.a.benninga@amc.uva.nl 